\title{
LA DIGNIDAD HUMANA: ENTRE EL DERECHO Y LA MORAL
}

\author{
Elena Beltrán \\ Universidad Autónoma de Madrid
}

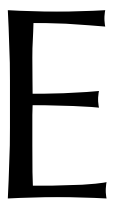

n la obra de Liborio HIERRO y de Francisco LAPORTA es fácil encontrar muchos temas y problemas de primer orden dentro de la filosofía jurídica magníficamente tratados. Por ello, la Filosofía jurídica en español tiene una deuda impagable con ellos. La obra de ambos autores brinda grandes posibilidades de encontrar puntos de interés y de debate sobre los que podría centrarse este breve texto. Sin embargo, de todo el abanico de posibilidades para articular una contribución a este merecido homenaje, voy a elegir un tema menor en la obra de ambos autores, que podríamos entender que está implícito en gran parte de sus temas, pero que en muy pocas ocasiones se hace explícito. Me refiero al tema de la dignidad humana. Es un tema complejo y muy presente en las declaraciones de derechos y en la jurisprudencia, no tanto en las reflexiones teóricas, por ello creo que entablar un diálogo en torno al mismo puede tener interés.

Conocemos las excelentes páginas que HIERRO y LAPORTA han dedicado a los derechos humanos, también conocemos que en las declaraciones de derechos más influyentes y más relevantes aparece de modo destacado alguna alusión a la idea de dignidad humana, de manera que derechos humanos y dignidad humana parecen ir de la mano en el imaginario más habitual ${ }^{1}$. Aunque esto no es algo que se refleje a primera vista en los textos de los autores mencionados. Y de sus referencias a la idea de dignidad se desprenden algunos sobreentendidos y algunos interrogantes.

En el caso de Liborio HIERRO, aparecen alusiones a la dignidad humana vinculadas a la propuesta de Ronald DWORKIN, de la «vaga pero poderosa» idea de dignidad humana como el especial valor moral de que están dotados los seres humanos. Ese valor moral esencial está vinculado, nos dice HIERRO, a la posibilidad de elegir los propios fines (autonomía); llevarlos a cabo (libertad); y asumir las consecuencias $(\text { responsabilidad })^{2}$.

La idea de dignidad humana en la obra de LAPORTA no aparece, o no la he encontrado, explícitamente mencionada en sus textos sobre derechos humanos sino en el capítulo primero de su libro sobre el imperio de la ley. Ahí plantea LAPORTA la

1 El Preámbulo del Pacto Internacional de los Derechos Civiles dice que los derechos humanos derivan de la dignidad inherente a los seres humanos. La Convención de Ginebra también habla de dignidad personal, de trato digno, y así muchos otros textos y declaraciones.

2 L. HiERRO, Los derechos bumanos. Una concepción de la justicia, 2016, cap. III, 133; cap. IV, 139. 
concepción de autonomía que fundamenta su construcción de imperio de la ley. En el último punto del capítulo, antes de las precisiones finales, LAPORTA se apoya en PiCO DELLA MiRANDOla para mencionar una idea de dignidad humana que conduce a una idea de autonomía personal, de las personas como seres que se hacen a sí mismos «no es solo que boy pueda yo prever un deseo o un interés de mi yo futuro y obrar en consecuencia para satisfacerlo, es que puedo ver y considerar qué clase de persona quiero ser y proceder paso a paso a constituir esa clase de persona» ${ }^{3}$. Pone el énfasis LAPORTA en la idea de los planes de vida como elemento esencial de la autonomía personal, en la capacidad de control de esos planes. La autonomía personal se presenta como un valor intrínseco vinculado inextricablemente a la idea de dignidad personal ${ }^{4}$.

\section{LA «VAGA Y PODEROSA» IDEA DE DIGNIDAD}

Es posible que la razón de la ausencia de la idea de dignidad en los textos de LAPORTA y de HIERRO tenga que ver con los problemas que plantea esta idea y las cargas semánticas tan fuertes y a la vez contradictorias que acarrea. Está claro que no siempre se ha entendido la idea de dignidad del mismo modo, y a día de hoy, todavía encontramos divergencias en cuanto al significado de la misma. La idea que parece abrirse paso en paralelo al desarrollo de los derechos humanos es una concepción de la dignidad que se entiende como inherente al hecho de ser persona, que no se pierde ni siquiera cuando se actúa inmoralmente porque es un atributo de los seres que pertenecen al reino de los fines.

En esta primera aproximación a la idea de dignidad saltan a la vista algunas dualidades esenciales. De un lado, las declaraciones de derechos nos hablan de la dignidad inherente a los seres humanos a la vez que nos conminan a hacer todo lo posible para garantizar la dignidad de todas las personas. Stephen PINKER nos dice que «leemos que la esclavitud y la degradación son moralmente erróneas porque arrebatan la dignidad. Pero también leemos que nada que se haga a una persona, incluyendo su esclavitud o degradación puede arrebatarle su dignidad» ${ }^{5}$. Otra de las dualidades de la idea de dignidad estriba en considerarla la base de los derechos o el contenido de los mismos.

Históricamente la dignidad era un predicado que diferenciaba, destacaba a algunos, no se atribuía por igual a los seres humanos. Consistía en una idea de respeto asociada a una excelencia o virtud de algún tipo, por nacimiento o merecimiento. Dignidad era un término de separación, de jerarquización. Era un término social, dentro de una constelación de valores y virtudes morales. El cristianismo igualó a todas las personas en la consideración de hijos de Dios. Sin embargo, esa igualdad derivaba del respeto a las leyes procedentes de la divinidad. En los escritos de los teólogos católicos se pone mucho énfasis en la idea de dignidad como valor intrínseco. Así, TOMÁs DE

3 F. LAPORTA, El imperio de la ley: una visión actual, 2007, 31, cursivas en el original.

4 LAPORTA, op. cit., 35.

5 S. PINKER, «The Stupidity of Dignity»; New Republic, 28 de marzo de 2008, citado en J. WALDRON, «Dignity and Rank», en Dignity, Rank and Rights, Tanner Lectures on Human Values, 2009, 211, nota 5. 
AQUiNo deja claro en sus escritos que la dignidad es el valor de ocupar el lugar que a cada uno le corresponde dentro del diseño que Dios hizo en la creación y revelado por las Escrituras y por la ley natural. Este discurso de la dignidad como valor intrínseco permea toda la doctrina de la iglesia católica y está claramente vinculada a la desigualdad y al respeto a la jerarquía, teológica, eclesiástica y social, así se refleja con claridad en lo que se refiere a los roles de las mujeres, la asunción de un rol revestido de un discurso de excelencia que es en realidad subordinación, será lo que confiera a las mujeres su dignidad, su valor intrínseco como seres humanos ${ }^{6}$.

El imperativo categórico kantiano define los límites de una esfera que ha de quedar fuera del alcance de los otros. La dignidad infinita de cada persona exige que los demás respeten la inviolabilidad de esa esfera de voluntad libre. El valor absoluto inherente a nuestra personalidad moral se configura como la base de nuestra autoestima, a la vez que es el pilar de la exigencia a los demás del respeto hacia uno mismo y la base de la igualdad entre todos.

El desafío consiste en superar la gran paradoja que nos plantean los ilustrados y los epígonos de la ilustración con la generalización de un concepto de dignidad procedente de las diferenciaciones de estatus de las sociedades jerárquicas, con la finalidad de igualar el estatus de las personas y de universalizar esa igualdad.

Podríamos interpretar con HABERMAS, que la nueva categoría de los derechos humanos reunifica dos elementos que se habían separado antes, en la desintegración del derecho natural cristiano y que se desarrollaron posteriormente en direcciones opuestas. Por un lado, la moral internalizada y justificada racionalmente, anclada en la conciencia individual, de cuño kantiano; por otro lado, los derechos positivos, promulgados, coactivos, que asientan las bases de las instituciones del estado moderno y de la sociedad de mercado. La idea de dignidad humana, para HABERMAS, se convierte en el eje conceptual que permite hacer la conexión entre estos dos elementos. Para llegar a este punto es necesario partir del medievo, de la individualización de los seres humanos, creados a imagen y semejanza de Dios y enfrentados a un juicio final que juzgará sus acciones como personas únicas e irreemplazables. Es el primer paso para un proceso que tiene un hito fundamental en la escolástica española y la subjetivación de los derechos naturales por contraposición al derecho natural objetivo. GROCIO y PUFENDORF son peldaños necesarios, KANT culmina este camino $^{7}$.

O tal vez el modo de lograr esa superación o ese puente que busca HABERMAS nos lo brinde el mismo KANT, como ocurre en la lectura e interpretación del mismo que presenta Stephen DARWALL, cuando encuentra en este autor una concepción de dignidad que tiene más que ver con la forma en que exigimos respeto de los demás a través de las demandas de la «segunda persona» que con una noción de valor inapreciable de nuestra capacidad moral.

6 M. Rosen, «Reply to Jeremy Waldron», en J. Waldron, Dignity, Rank and Rights, 2012, 9, en donde menciona que estos discursos se encuentran en el fundamento del rechazo de muchos igualitaristas que conocen la tradición católica a la asunción de la idea de dignidad como idea clave.

7 J. HABERMAs, El concepto de dignidad humana y la utopía realista de los derechos bumanos, 2010, 3-25. 


\section{DE LA DIGNIDAD COMO VALOR A LA DIGNIDAD COMO ESTATUS}

La propuesta de Jeremy WALDRON de construir la idea de dignidad desde el propio marco jurídico, como un concepto iusfilosófico presenta ventajas interesantes en orden a una clarificación de un término con un especial relieve en los textos jurídicos y en las decisiones jurisprudenciales. De la necesidad de esta clarificación da cuenta la escandalizada cita de WALDRON en relación con la jurisprudencia del Tribunal Supremo canadiense que decide en 2008 abandonar la idea de dignidad humana como referencia en su doctrina antidiscriminatoria por encontrarla «abstracta», «subjetiva», «confusa y difícil de aplicar» ${ }^{8}$.

La definición de dignidad de WALDRON, que fundamenta su interesante propuesta, nos dice que se trata de «un término usado para indicar el rango más alto, jurídico, político y social, y la idea de dignidad humana sería la asignación del más alto rango de estatus a todas las personas»? . Entendiendo, con John Austin, que cuando hablamos de estatus hacemos una elipsis para indicar un conjunto de derechos y deberes que están juntos por una razón subyacente que explica el conjunto y le da coherencia. Así, por ejemplo, el estatus de niño o de extranjero y el conjunto de derechos, obligaciones, deberes, asociados al mismo. Hemos de entender también, que la posición jurídica de niño, como la de extranjero, nunca está del todo cerrada, porque pueden presentarse nuevos incidentes o desaparecer los antiguos ${ }^{10}$. Por ello, la idea de dignidad como estatus va acompañada de una cierta flexibilidad.

Las declaraciones de derechos sugieren que la dignidad es la base de los derechos, pues los derechos «derivan de la dignidad inherente a las personas» ${ }^{11}$. La pregunta de WALDRON es si esto supone que el ideal moral de dignidad es un fundamento extralegal de los derechos humanos. Su respuesta pretende mostrarnos que no ha de serlo necesariamente. Para ello acude como hemos visto a la construcción de una idea de dignidad como estatus. Las implicaciones de esta propuesta van en la línea de articular una construcción jurídica y política de la dignidad en unos términos similares a los que llevan a la construcción de la idea de ciudadanía.

La dignidad dejaría de ser el objetivo o telos de los derechos humanos, sería un estatus normativo y muchos derechos humanos habrían de entenderse como incidentes de ese estatus. Si se configura la dignidad de este modo, existiría todavía una dualidad entre las normas generales que establecen ese estatus y las normas particulares que prohíben su degradación pero ya no sería la misma relación entre un objetivo y los principios subordinados que promueven ese objetivo ${ }^{12}$.

No parece casual que WALDRON acuda a FULLER y a la idea de moralidad interna del derecho cuando defiende su idea de dignidad y enumera como ha de ser sustentada en los diferentes elementos esenciales de un ordenamiento jurídico, a saber: en la idea de autoaplicación, como el uso de reglas que implica la idea de contención o autocon-

\footnotetext{
${ }^{8}$ R. v Kapp, [2008] SCR 41, at sec. 22.

9 WALDRON, op. cit., 233.

10 J. Austin, Lectures of Jurisprudence or The Philosophy of Positive Law, citado en WALDRON, 242,

${ }_{11}$ Declaración de Derechos Civiles y Políticos.

12 J. WALDRON, 2009, 212.
} nota 112 . 
trol; en el uso de estándares; en las necesarias audiencias; en la argumentación; en la representación; o, finalmente en la coacción ${ }^{13}$.

Sin embargo, lo fundamental en la propuesta de WALDRON es el elemento central sobre el que pivota su construcción de la idea de dignidad, porque se trata fundamentalmente de igualdad, de asumir un estatus que otorga un rango superior a las personas, que es el rango superior al que da acceso el reconocimiento de los derechos humanos, la novedad con respecto al significado tradicional de dignidad es que ese rango se otorga a todas las personas por igual.

\section{IGUALDAD, DIGNIDAD Y DERECHOS HUMANOS: COMENTARIOS Y PREGUNTAS}

En las citas de HIERRO y de LAPORTA mencionadas al principio, la idea de dignidad humana se nos presenta aparentemente como una idea moral inextricablemente unida a la idea de autonomía y fundamento de los derechos humanos, en definitiva, de una idea de dignidad como valor en el sentido habitualmente atribuido a KANT. Todo parece indicar que podríamos pensar en una interpretación en esa línea. Sin embargo, las referencias de las que parten ambos autores, aunque completamente diferentes entre sí, nos podrían llevar más cerca de la propuesta de WALDRON de lo que nos muestran las apariencias.

En el caso de Liborio HIERRO la vinculación de su idea a la concepción de dignidad que atribuye a Ronald DwORKIN nos presenta una idea de dignidad asociada a la autonomía, la libertad y la responsabilidad de los seres humanos con su propia vida. Es interesante la inclusión de la idea de responsabilidad, pues sugiere una concepción de autonomía matizada, no solo vinculada a la libertad de elección ${ }^{14}$. No menciona HiERro la idea de igualdad, sin embargo, sí parece estar esta idea en alguna medida en las propuestas de DwORKIN. Basta recordar la relevancia de la idea de igualdad en las primeras formulaciones de su teoría, desde Taking Rights Seriously, cuando consideraba el eje de sus posiciones la idea de igualdad en contraposición a las ideas que denominaba kantianas, que incluían seguramente las ideas de dignidad como valor moral absoluto que hemos mencionado ${ }^{15}$.

Si pensamos en las alusiones a la idea de dignidad en los textos de LAPORTA encontramos a PICO DE LA MiRANDOLA, pero también una sutil presencia de FULLER, como por otra parte, en todo el planteamiento de su idea de imperio de la ley ${ }^{16}$. En el caso de PICO DE LA MiRANDOLA, no faltaría quien defienda que podría ser interpretado en un sentido más cercano a la propuesta de WALDRON. La influencia de FULLER es, en mi opinión, una influencia compartida de WALDRON con LAPORTA. Es interesante también contrastar la idea de dignidad como estatus que presenta WALDRON con la idea de igualdad de LAPORTA. Encaja en muchos puntos. No es posible entrar aquí en más detalles.

\footnotetext{
13 WaLdRON, op. cit., 237-253; L. FULlER, The Morality of Law, 1964, cap. 2.

14 WALDRON, op. cit., 141-142.

15 DwORKIN, Taking Rights Seriously, 1977.

16 Fuller, The Morality of Law, 162.
} 
No sé si HIERRO y LAPORTA suscribirían una interpretación de su idea de dignidad en los términos que he presentado en estas páginas, un tanto apresuradas, pero la vinculación de la dignidad a la igualdad me parece que insiste en la construcción de una mejor idea de derechos humanos. De manera que la idea de dignidad como estatus sería muy compatible con las concepciones de derechos defendidas por ambos autores. No se trata de colocar esta idea de dignidad como estatus en la categoría de fundamento único de los derechos humanos, sino de situarla entre una de las fundamentaciones posibles de los mismos.

No quiero terminar sin decir que los años compartidos con Liborio HIERRO y Francisco LAPORTA en la Universidad Autónoma han sido un privilegio y es difícil poner en palabras lo que han significado en mi formación intelectual.

\section{REFERENCIAS BIBLIOGRÁFICAS}

Dworkin, R., 1977: Taking Rights Seriously, Harvard University Press. Trad. Tomando los derechos en serio, Barcelona: Ariel.

- 2006: Is Democracy Possible Here? Principles for a New Political Debate, Princeton University Press. Trad. La democracia posible, Barcelona: Paidós.

Fuller, L., 1977: The Morality of Law, Yale University Press [1964].

HABERMAS, J., 2010: «El concepto de dignidad humana y la utopía realista de los derechos humanos», en Dianoia, vol. LV, 64, mayo, 3-25.

Hierro, L., 2016: Los derechos humanos. Una concepción de la justicia, Madrid: Marcial Pons.

LAPORTA, F., 2007: El imperio de la ley: una visión actual, Madrid: Trotta.

Waldron, J., 2012: Dignity, Rank and Rights, Oxford University Press (las citas de este texto son de una versión en $p d f$ de las Tanner Lectures on Human Values). 